\title{
CONSTRUCCIONES DE PARTICIPIO Y DE INFINITIVO EN TEXTOS ESPAÑOLES MEDIEVALES Y PRECLÁSICOS
}

El presente estudio se dedica a dos fenómenos que afectan a las construcciones medievales españolas formadas por un verbo flexionado y un participio o un infinitivo dependiente: la anteposición del participio y del infinitivo al verbo regente y la interpolación de constituyentes entre las dos formas verbales que componen los complejos verbales en cuestión. Tales fenómenos, examinados desde el punto de vista estructural en el estudio precedente de este mismo volumen, habituales en las variedades medievales de todas las lenguas romances de la Península Ibérica, aparecen sólo esporádicamente y casi siempre como alternativas sintácticas marcadas o arcaizantes. Los ejemplos que se aducen en (1-2) ilustran la anteposición del participio y del infinitivo, normal en el español antiguo, frente a los ejemplos de (3-4), los cuales demuestran que tal orden no es natural en el español actual ni con participio ni con infinitivo.
a. esp. med.
cogida han la tienda do albergaron de noch (Cid, 2706)
b. esp. precl. como dicho es (CronEsp, 19v)
a. esp. med.
Ond $<$ e $>$ auenir suele $\mathrm{q}<\mathrm{ue}>$ (FueJuz, 10v)
b. esp. med.
ca departir vos quiero yo lo que [...] (VeiRey, 8r)
a. esp. act.
Mi hermano ha leído el libro
b. esp. act.
*Leído el libro ha mi hermano
a. esp. act.
b. esp. act.
Mi hermano quiere leer el libro
*Leer el libro mi hermano quiere

Los ejemplos medievales que se aducen en (5-6) demuestran que en las construcciones medievales de ambos tipos, al lado de la adyacencia de las dos formas verbales - (5a) y (6a)-, era posible intercalar diferentes constituyentes oracionales entre ellas, como se ilustra en (5b-e) y (6b-e) en construcciones de participio y de infinitivo, respectivamente. (Las formas verbales de las construcciones aparecen en cursiva, los constituyentes interpolados se destacan en negrita). Los ejemplos modernos de (7) prueban que en el español actual con el participio sólo es correcta la adyacencia de los dos verbos. Aunque Suñer ${ }^{1}$ y Andrés-Suárez aducen ejemplos modernos de interpolación, como dice esta última autora, esta alternativa "se siente como afectada" y solamente "es utilizada por algunos escritores de gustos arcaizantes" ${ }^{2}$. Los ejemplos modernos de (8) demuestran que también en el caso de las construcciones de infinitivo se ha restringido el número de constituyentes que se pueden intercalar: (8b), (8c) y (8d) -respectivamente- indican que la interpolación del complemento regido por el verbo dependiente no es posible, la del sujeto es dudosa y sólo la de los elementos adverbiales es aceptable.

\footnotetext{
${ }^{1}$ Margarita SUÑER: "Haber + Past Participle”, in: Linguistic Inquiry XVIII/4, 1987, págs. 683-684.

${ }^{2}$ Irene ANDRES-SUÁREZ, El verbo español. Sistemas medievales y sistema clásico. Madrid, Gredos, 1994, p. 63.
} 
(5)
a. esp. med.
los canonigos del sepulcro auien tenido aquel
b. esp. med.
logar gra $<\mathrm{n}>$ t tie $<\mathrm{m}>$ po. $(\mathrm{Gcu}, 14 \mathrm{v})$
c. esp. med.
ouieran al legado sacado de la cipdat a grant desondra (ConUlt, 7v)
$\begin{array}{ll}\text { c. } & \text { esp. med } \\ \text { d. } & \text { esp. med }\end{array}$
Despues que la carta ouo el moro fecha (InfLara,
$\begin{array}{ll}\text { d. } & \text { esp. med. }\end{array}$
$34 \mathrm{v})$
$\&$ bien cuydo que so ende muerto (InfLara, 32v) quando ovo el conde el agua atravesado (Fn. Gonz., 357d, citado por Andres-Suárez, op. cit.)

(6)

$\begin{array}{ll}\text { a. } & \text { esp. med. } \\ \text { b. } & \text { esp. med. } \\ \text { c. } & \text { esp. med. } \\ \text { d. } & \text { esp. med. } \\ \text { e. } & \text { esp. med. }\end{array}$

assi $c 0<\mathrm{m}>$ mo llegaron: quisieron mostrar sus bondades (ConUlt, 2r)

el ioyz deue las testimonias reçebir. (FueJuz, 15r) la partida $\mathrm{q}<\mathrm{ue}>$ deue $<n>$ amas las partes auer (FueJuz, 15v)

$\& \mathrm{q}<$ ue $>$ podam $<$ os $>$ firme mient $<$ re $>$ defender $\mathrm{s}<\mathrm{us}>$ pueblos. (FueJuz, 10v)

(7)

$\begin{array}{ll}\text { a. } & \text { esp. act. } \\ \text { b. } & \text { esp. act. } \\ \text { c. } & \text { esp. act. } \\ \text { d. } & \text { esp. act. }\end{array}$
quel enuiaua el Rey mucho saludar. co $<\mathrm{m}>\mathrm{mo}$ amigo. (ConUlt, 10v)

$\begin{array}{ll}\text { a. } & \text { esp. act. } \\ \text { b. } & \text { esp. act. } \\ \text { c. } & \text { esp. act. } \\ \text { d. } & \text { esp. act. }\end{array}$

Mi hermano ha leído el libro

* Me hermano ha el libro leído

*Ha mi hermano leído el libro

*Mi hermano ha esta semana leído el libro

Anteriormente ya se han explicado detalladamente los argumentos que apoyan una hipótesis que supone que los paralelos observables en la "superficie" de la evolución de las dos construcciones se deben a los mismos cambios en lo profundo de las estucturas. Esta vez me limito a señalar que las diferencias entre la variedad medieval y la variedad actual del español están en relación con algunos cambios producidos en las reglas de la colocación de los constituyentes regidos por el verbo, ocurridos después de la Edad Media. En concreto, se trata de que en el español medieval tanto la anteposición de los verbos no flexionados, como la interpolación de constituyentes entre ellos eran posibles gracias al sistema conocido por el nombre V2 de la sintaxis medieval, el cual permitía que el verbo fuese precedido por cualquier constituyente, incluso por sus propios argumentos. Después de la Edad Media este sistema fue sustituido por el nuevo orden tipo SVO, que exige que los constituyentes regidos por el verbo se coloquen a su derecha: así el verbo flexionado debe ser seguido por el verbo no flexionado y éste, por su propio complemento.

Basándome en la idea de paralelos en la evolución de los dos tipos de construcciones, he realizado el análisis de la frecuencia de la anteposición del verbo 
complemento y de la interpolación de constituyentes entre las formas verbales en construcciones de participio y de infinitivo. Otros trabajos, concentrados en una de las dos construcciones, ya habían ofrecido datos estadísticos referentes a estos fenómenos. En el artículo de Company ${ }^{3}$, dedicado a la evolución de las formas verbales compuestas formadas por haber o ser más participio, el orden de los constituyentes es uno de los temas centrales. Sus datos fueron citados y enriquecidos posterioremente por Andres-Suárez ${ }^{4}$ y García Martín ${ }^{5}$. La interpolación de constituyentes y parcialmente la anteposición del verbo complemento fueron examinados como posibles factores diferenciadores en la evolución de la subida de clíticos en las construcciones formadas por un verbo regente $\mathrm{y}$ un infinitivo dependiente en varios trabajos míos consagrados a las construcciones de infinitivo del español y del portugués ${ }^{6}$. La existencia de los paralelos arriba mencionados, interpretables como manifestaciones de los mismos fenómenos estructurales, sin embargo, me motivó a investigar las construcciones de participio y de infinitivo en conjunto, con los mismos criterios. Los objetivos múltiples y complejos propuestos en las investigaciones eran los siguientes: de un lado, examinar la frecuencia de la anteposición y de la interpolación en las construcciones de participio y en las construcciones de infinitivo en conjunto en el mismo corpus para poder comparar las dos construcciones; de otro lado, estudiar la frecuencia de los fenómenos en cuestión en textos medievales y en textos del período preclásico con el fin de poder extraer conclusiones diacrónicas; finalmente analizar la frecuencia de frecuencia en textos de diferentes géneros para averiguar si había diferencias en la distribución de los dos fenómenos según tipos de textos o según estilos.

Para que el corpus pudiese corresponder a los objetivos de la investigación, al establecerlo se ha tenido en cuenta

a) que los textos perteneciesen a tres géneros distintos: crónicas, textos legales y obras literarias;

b) que la datación de la versión utilizada del texto no fuese muy posterior a la fecha de nacimiento del original;

c) que todos los textos fuesen escritos en prosa.

El corpus creado con estos criterios está constituido por los siguientes textos: un fragmento de unas 10 mil palabras de la versión leonesa del Fuero Juzgo (conservado en una copia del siglo XIII); un fragmento de unas 11 mil palabras de la Gran Conquista de Ultramar (escrita en el siglo XIII y conservada en una copia del XIV); la leyenda de los Siete Infantes de Lara, reproducida en forma prosificada en la Crónica de Veinte Reyes (conservada en una copia del siglo XIV o XV); un

\footnotetext{
${ }^{3}$ Concepción COMPANY, "Sintaxis y valores de los tiempos compuestos en el español medieval”, in: Nueva Revista de Filología Hispánica, XXXII/2, 1983, págs. 235-257.

${ }^{4}$ Irene ANDRES-SUÁREZ, op. cit.

5 José María GARCÍA MARTÍN, La formación de los tiempos compuestos del verbo en español medieval y clásico. Aspectos fonológicos, morfológicos y sintácticos. Valencia, Universitat de València, 2001.

${ }^{6}$ Tibor BERTA, "La estructura de las construcciones de infinitivo en textos españoles y portugueses medievales", in: Sándor KISS- Giampaolo SALVI-Ildikó SZIJJ (eds.), rom.hu. Études Romanes de Budapest, 3. Budapest, Programme Doctoral de Linguistique Romane de l'Université Eötvös Loránd/Íbisz, 2001, 33-47. Idem, Clíticos e infinitivo. Contribución a la historia de la promoción de clíticos en español y portugués. Szeged, Hispánia, 2003.
} 
fragmento de unas 24 mil palabras de la Crónica de España de Diego de Valera (editada en 1482); todo el texto de las Ordenanzas de Sevilla, promulgadas por los Reyes Católicos el 30 de mayo de 1492, (15.500 palabras); todo el texto de la Historia de Pierres y Magalona (versión española del siglo de principios del siglo XV, de autor anónimo), (23 mil palabras); el primer acto de La Celestina según el manuscrito 1520 de la Biblioteca de Palacio de Madrid (siglo XVI) (4.500 palabras) ${ }^{7}$. En cuanto a las ocurrencias que se han tenido en cuenta se debe adelantar que se han excluido del análisis aquellas construcciones en las que el participio o el infinitivo, respectivamente, desempeña función adverbial, es decir, no depende estrictamente del verbo flexionado. De entre los casos de interpolación se han excluido aquellas construcciones de participio con ser en las que el participio podía ser interpretado como adjetivo y no como verbo.

El Cuadro 2.1. resume los datos del corpus español seleccionado.

\begin{tabular}{|l|r|r|r|}
\hline \multicolumn{1}{|c|}{ Textos } & \multicolumn{1}{c|}{ Palabras } & \multicolumn{1}{c|}{ Inf } & \multicolumn{1}{c|}{ Part } \\
\hline ConUlt & 10.900 & 297 & 159 \\
\hline FueJuz & 9.700 & 313 & 89 \\
\hline InfLara & 6.900 & 103 & 33 \\
\hline CroEsp & 24.000 & 158 & 194 \\
\hline OrdSev & 15.500 & 208 & 175 \\
\hline PieMag & 23.000 & 471 & 343 \\
\hline Celestina & 4.500 & 56 & 25 \\
\hline Total & 94.500 & 1.606 & 1.018 \\
\hline
\end{tabular}

Cuadro 2.1. Constitución del corpus español examinado

\section{Proporción de la anteposición}

El primer fenómeno analizado ha sido el de la anteposición del verbo en forma de participio o infinitivo, ya ilustrado con los ejemplos de (1) y (2). El Cuadro 2.2. resume los datos referentes a la proporción de la anteposición del participio al verbo regente -haber o ser- en los textos analizados, mientras que el Cuadro 2.3. presenta la frecuencia del mismo fenómeno en las construcciones de infinitivo.

\footnotetext{
${ }^{7}$ Los datos bibliográficos del corpus -con las siglas utilizadas en este trabajo- son los siguientes: Admyte, 1995=Archivo Digital de Manuscritos y Textos Españoles, Madrid, Micronet, 1995; ConUlt=Gran Conquista de Ultramar, in: Admyte, 1995; CroEsp=Diego de VALERA, Crónica de España (1482), in: Admyte, 1995; FueJuz=Fuero Juzgo, in: Admyte, 1995; InfLara=Leyenda de los siete infantes de Lara, in: VeiRey, Admyte, 1995; OrdSev=Ordenanzas de Sevilla (1492), in: Admyte, 1995; PieMag=Historia de Pierres y Magalona, in: Admyte, 1995; VeiRei=Crónica de veinte reyes, in: Admyte, 1995; Celestina=Fernando de ROJAS, Tragicomedia de Calisto y Melibea, manuscrito 1520 de la Biblioteca de Palacio, Madrid. Transcripción de Charles FAUBER, www.cervantesvirtual.com/bibl_obra/celestina
} 


\begin{tabular}{|l|r|r|r|}
\hline \multicolumn{1}{|c|}{ Textos } & \multicolumn{1}{c|}{ VPart } & \multicolumn{1}{c|}{ PartV } & \multicolumn{1}{c|}{ Total } \\
\hline FueJuz & 89 & 0 & 89 \\
\hline ConUlt & 159 & 0 & 159 \\
\hline InfLar & 32 & 1 & 33 \\
\hline CronEsp & 192 & 2 & 194 \\
\hline OrdSev & 175 & 0 & 175 \\
\hline PieMag & 343 & 0 & 343 \\
\hline Celestina & 23 & 2 & 25 \\
\hline Total & 1.013 & 5 & 1.018 \\
\hline
\end{tabular}

Cuadro 2.2. Frecuencia del participio antepuesto

\begin{tabular}{|l|r|r|r|}
\hline \multicolumn{1}{|c|}{ Textos } & \multicolumn{1}{c|}{ VInf } & \multicolumn{1}{c|}{ InfV } & \multicolumn{1}{c|}{ Total } \\
\hline FueJuz & 312 & 1 & 313 \\
\hline ConUlt & 295 & 2 & 297 \\
\hline InfLar & 102 & 1 & 103 \\
\hline CronEsp & 153 & 5 & 158 \\
\hline OrdSev & 207 & 1 & 208 \\
\hline PieMag & 471 & 0 & 471 \\
\hline Celestina & 52 & 4 & 56 \\
\hline Total & 1.592 & 14 & 1.606 \\
\hline
\end{tabular}

Cuadro 2.3. Frecuencia del infinitivo antepuesto

Se puede ver que la frecuencia de este fenómeno es muy baja en los textos analizados en ambos tipos de construcciones. Se han recogido 5 casos con participio antepuesto al verbo regente, cifra que apenas llega al 0,5 por 100 del total de las construcciones de participio. El único caso medieval de participio antepuesto se presenta en (9), mientras que los casos preclásicos en (10).

com $<\mathrm{m}>$ o cuydades que olujdado auia la desonrra $\mathrm{q}<\mathrm{ue}>\mathrm{me}$ fezistes en burgos (InfLara, 37r)

(10) a. si culpado no es (CroEsp, 8v)

b. $\quad$ como dicho es (CroEsp, 19v)

c. $\quad$ S. señor luego fecho es (Celestina, $94 \mathrm{v}$ )

d. que oydo he dez'ir q[*ue] (Celestina, 95r)

Como demuestran los ejemplos aducidos, la anteposición se documenta tanto con el verbo regente haber como con ser. Nótese que en el caso presentado en (9) sería normal la concordancia del participio con el objeto directo -la desonrra-, no obstante tal concordancia no se establece, el participio aparece en forma invariable-olujdado-.

En los textos analizados también es muy baja la frecuencia de aquellas construcciones en las que el infinitivo dependiente se le antepone al verbo regente: esta estructura apenas alcanza el 0,9 por 100 del total de los ejemplos españoles registrados 
que contienen infinitivos dependientes, pues se han recogido tan sólo 14 casos de anteposición frente a 1592 casos de orden VInf. Los casos con infinitivos antepuestos procedentes del período medieval se presentan en (11), mientras que los casos que ilustran el mismo fenómeno en textos preclásicos son los que se aducen en (12).

Ond $<\mathrm{e}>$ auenir suele $\mathrm{q}<\mathrm{ue}>$ mayor $\mathrm{p}<\mathrm{ro}>\mathrm{l}$ gana dela $\mathrm{sal}<\mathrm{u}>\mathrm{t}$ del $<$ os $>$ ot $<$ r $>0$ `s q $<$ ue $>$ dela suya. (FueJuz, 10v)

b. que darle querie guerra. (ConUlt, $21 \mathrm{v}$ )

c. desamparar no $<\mathrm{n}>$ los` querien (ConUlt, 24r)

d. $\quad$ ca nos toda via yr queremos con nuestro tio Ruy blasq $<$ ue $>$ s (InfLara, 35r)

(12) a. o atañer puede en qual quier manera (OrdSev, 2r)

b. E como esto con gran uoluntad conplir desease (CronEsp, 2r)

c. por que (...) vuestra real magestad en esto seruirle pudiese (CronEsp, 2r)

d. que $m a<n>$ dar no se pueden (CronEsp, 3r)

e. ala qual visitar me enbio el serenissimo Rey don Juan (CronEsp, 25v)

f. quien saber las querra ${ }^{8}$ (CronEsp, 27r)

g. que verte alcançase/ (Celestina, 94r)

h. $\quad y$ en tan convenjente lu[*gar] do mj secreto dolor magnifestar te pudies'e (Celestina, 94r)

i. $\quad$ que ver yo non pude (Celestina, 97v)

j. $\quad$ vella querias (Celestina, 98r)

Si comparamos los datos correspondientes a las dos épocas diferentes, también encontramos resultados interesantes, puesto que observamos que los casos de anteposición se limitan prácticamente a los textos de la época preclásica: de los 5 casos de participio antepuesto 4 se han detectado en los textos renacentistas, mientras que de los 14 infinitivos antepuestos 10 aparecen también en ellos. Estos datos confirman las conclusiones de las investigaciones de Company, según las cuales la anteposición del participio, frecuente en el siglo XII, es cada vez más rara a partir del siglo XIII, no obstante, reaparece en el siglo XV.

La comparación de la frecuencia del fenómeno en los diferentes tipos de textos indica que los rasgos característicos del género determinan en cierta manera su frecuencia. La proporción de la anteposición es muy baja en los textos legales: en este género sólo se han registrado 2 ocurrencias con infinitivo (una en el Fuero Juzgo y otra en las Ordenanzas de Sevilla) y ninguna con participio; en las crónicas y en las demás obras literarias la frecuencia de la anteposición es superior tanto con participio como con infinitivo, aunque su distribución en los textos preclásicos es desequilibrada. Casi todos los casos de anteposición aparecen en dos textos: de los 4 casos de participio antepuesto 2 se han registrado en la Crónica de España y 2 en la Celestina; los 10 casos de infinitivo antepuesto, registrados en la época preclásica, 9 se reparten entre los fragmentos analizados de la Crónica de España y La Celestina.

\footnotetext{
${ }^{8}$ En el texto se lee quien saber las guerra, pero probablemente se trata de una errata, y la $g$ se debe leer como $q$.
} 
Nótese que, de modo un tanto sorprendente, no se ha registrado ningún caso de anteposición -ni con participio ni con infinitivo- en la Historia de Pierres y Magalona, la otra obra literaria procedente de la misma época.

\section{Proporción de la interpolación}

La frecuencia de la interpolación de elementos entre el verbo flexionado y el participio se resume en el Cuadro 2.4, mientras que la frecuencia del mismo fenómeno entre el verbo flexionado y el infinitivo se puede ver en el Cuadro 2.5. En ambos casos se presentan los datos de interpolación según la categoría del constituyente interpolado. Nótese, sin embargo, que entre las cifras de interpolación no se han tenido en cuenta las preposiciones, que pueden intercalarse actualmente también y tampoco los pronombres clíticos, los cuales, como elementos inacentuados, se colocaban según reglas especiales.

\begin{tabular}{|c|c|c|c|c|c|c|}
\hline \multirow[t]{2}{*}{ Textos } & \multicolumn{4}{|c|}{ Int+ } & \multirow{2}{*}{ Int- } & \multirow{2}{*}{ Total } \\
\hline & Sujeto & Objeto & Adverbio & Int+ total & & \\
\hline FuеJuz & 2 & 0 & 8 & 10 & 79 & 89 \\
\hline ConUlt & 7 & 2 & 13 & 22 & 137 & 159 \\
\hline InfLara & 3 & 1 & 2 & 6 & 27 & 33 \\
\hline CronEsp & 6 & 0 & 45 & 51 & 143 & 194 \\
\hline OrdSev & 0 & 0 & 7 & 7 & 168 & 175 \\
\hline PieMag & 5 & 1 & 27 & 33 & 310 & 343 \\
\hline Celestina & 2 & 0 & 0 & 2 & 23 & 25 \\
\hline Total & 25 & 4 & 102 & 131 & 887 & 1.018 \\
\hline
\end{tabular}

Cuadro 2.4. Interpolación de constituyentes con participio

\begin{tabular}{|c|c|c|c|c|c|c|}
\hline Textos & \multicolumn{4}{|c|}{ Int+ } & Int- & Total \\
\hline & Sujeto & Objeto & Adverbio & Int+ total & & \\
\hline FиеJиz & 9 & 7 & 12 & 28 & 285 & 313 \\
\hline ConUlt & 17 & 3 & 29 & 49 & 248 & 297 \\
\hline InfLara & 6 & 2 & 10 & 18 & 85 & 103 \\
\hline CronEsp & 0 & 6 & 11 & 17 & 141 & 158 \\
\hline OrdSev & 0 & 0 & 7 & 7 & 201 & 208 \\
\hline PieMag & 7 & 3 & 65 & 75 & 396 & 471 \\
\hline Celestina & 3 & 3 & 3 & 9 & 47 & 56 \\
\hline Total & 42 & 24 & 137 & 203 & 1.403 & 1.606 \\
\hline
\end{tabular}

Cuadro 2.5. Interpolación de constituyentes con infinitivo

Se puede ver que la frecuencia del fenómeno también es parecida en el caso de ambos tipos de construcciones: los 131 casos de interpolación de constituyentes entre el verbo flexionado y el participio forman así el $13 \%$ de los 1018 ejemplos de 
construcciones con participio, mientras que los 203 casos de interpolación alcanzan el $12,6 \%$ de las 1606 construcciones de infinitivo.

No hay diferencia considerable entre la frecuencia del fenómeno en los textos pertenecientes a las dos épocas. En los textos medievales se han recogido 38 casos de interpolación, es decir, el 13,5\% de los 281 ejemplos de construcciones de participio, y en los textos preclásicos los 93 casos de interpolación constituyen el 12,6\% de las 737 ocurrencias. Por lo que se refiere a las construcciones de infinitivo, en los textos medievales se han registrado 95 ejemplos con constituyentes intercalados entre el verbo regente y el infinitivo; esta cantidad forma el 13\% de las 713 construcciones de infinitivo medievales. En los textos preclásicos se han recogido 108 casos de interpolación con infinitivo, que constituyen el $12 \%$ de los 893 ejemplos de construcciones de infinitivo preclásicas.

La distribución de los casos de interpolación según los tipos de textos muestra proporciones semejantes a las que hemos visto en el caso de la anteposición. La frecuencia de la interpolación es notablemente inferior en el caso de los textos legales: llega al $11 \%$ con participio y $9 \%$ con infinitivo en el Fuero Juzgo, mientras que alcanza el $4 \%$ con participio y el $3 \%$ en las Ordenanzas, respectivamente. En los textos medievales no legales los datos de frecuencia son los siguientes: en la Gran Conquista de Ultramar son del 14 y del 16,5\%, en la Leyenda de los Siete Infantes del 18 y del 17,5 \%, respectivamente. En los textos no legales preclásicos encontramos los datos siguientes: el 26 y el 10\% en la Crónica de España, el 9,6 y el $16 \%$ en la Historia de Pierres y Magalona, el 8 y el $16 \%$ en el acto primero de La Celestina.

También merece la pena observar la proporción de los constituyentes interpolados según su categoría: se puede ver que los elementos que con mayor frecuencia separan el verbo flexionado y el verbo dependiente son los que tienen función adverbial en ambos tipos de construcciones. En el caso de las construcciones de participio 102 veces se intercalan elementos adverbiales; los casos medievales se ven en (13-15), los casos preclásicos en (15-18).

(13) a. assi como es $\mathrm{d}<\mathrm{e}>$ suso dicho. (FueJuz, 11r)

b. $\quad \mathrm{q}<\mathrm{ue}>$ son bie $<\mathrm{n}>$ enmendadas $\&$ ordenadas. (FueJuz, 11v)

c. assi como es de suso dicho. (FueJuz, 13v)

d. $\quad$ los pleitos $\mathrm{q}<\mathrm{ue}>$ son ya començados \& $\mathrm{n}<\mathrm{on}>$ son acabados (FueJuz, 13r)

e. $\quad \& 1<0$ s $>$ pleitos $\mathrm{q}<\mathrm{ue}>$ son ya acabados antes $\mathrm{q}<\mathrm{ue}>$ estas leyes fuessen emendadas (FueJuz, 13r)

f. $\quad$ q $<$ ue $>$ n $<$ on $>$ sea $<$ n $>$ mas dema $<$ n $>$ dados (Fuejuz, 13r)

g. no $<$ n $>$ mandam $<$ os $>$ q $<$ ue $>$ en ne $<$ n $>$ guna manera sea $<$ n $>$ de cabo demandadas. (FueJuz, 13r)

h. $\quad \mathrm{q}<\mathrm{ue}>$ las partes no $<$ n $>$ sean mucho agrauiadas. (FueJuz, 14v)

(14) a. ca sos enemigos eran muy apremiados daquel logar (ConUlt, 13r)

b. por que los de dentro estaua $<\mathrm{n}>$ muy menguados de qua $<\mathrm{n}>\mathrm{t}\left({ }^{\wedge} \mathrm{o}\right)\left[{ }^{\wedge} \mathrm{a}\right] \mathrm{s}$ cosas auien mester. (ConUlt, $18 \mathrm{v}$ )

c. de $\mathrm{q}<\mathrm{ue}>$ muchas uezes auedes ya oydo. (ConUlt, $7 \mathrm{r}$ )

d. que fuera ya muchas uezes prouado en armas. (ConUlt, 13r) 
e. que era muy esforçado por que (ConUlt, 20v)

f. que fueron ende muy desmayados (ConUlt, 22v)

g. que eran muy aquexados de set. (ConUlt, 23v)

h. $\quad \operatorname{co}(\mathrm{n})[\mathrm{m}] \mathrm{mo}$ yuan tan bien acabdellados. (ConUlt, 25r)

i. $\quad$ co $<\mathrm{m}>$ mo auedes ya oydo. (ConUlt, 26v)

j. quando se uieron assi çercados (ConUlt, 13v)

k. $\quad$ qua $<$ n $>$ do se uiero $<$ n $>$ assi ap $<$ re $>$ miados de todas partes. (ConUlt, 14r)

1. $\mathrm{q}<\mathrm{ua}>\mathrm{n}<\mathrm{d}>\mathrm{o}$ se uieron $\tan$ cuyctados fiziero $<\mathrm{n}>$ lo assi. (ConUlt, 25v)

(15) a. \& bien cuydo que so ende muerto (InfLara, 32v)

b. de com $<m>$ o les aujan ya muertos a muño salido \& a ferrant gonçales su sobrino \& alos dozientos caualleros que (InfLara, 37r)

(16) a. les es aellos atribuydo \& denegado alos dichos notarios \& escriuanos (OrdSev, 2v)

b. el officio dela escriuania publica de Seuilla esta mal proueydo \& desordenado (OrdSev, 2v)

c. no van tanbien ordenadas \& actorizadas \& dignas de fe como se req $<$ u $>$ i'ere $($ OrdSev, 2v)

d. \& estos asy sacados sea $<$ n $>$ luego traydos \& presentados por los dichos alcaldes (OrdSev, 6v)

e. $\quad \&$ alli sea $<$ n $>$ luego examinados $($ OrdSev, 6v)

f. $\quad \&$ asi se esta iniusta mente detenido (OrdSev, 11v)

g. $\quad \&$ si asi no lo fiziere seye $<$ n $>$ do sobre ello req $<$ ue $>$ rido $\mathrm{q}<$ ue $>$ (OrdSev, 12v)

(17) a. si algun ladron es alli traydo (CroEsp, 8v)

b. donde Roma es agora situada (CroEsp, 28v)

c. La qual fue primero llamada dardania (CroEsp, 12v)

d. por lo qual fue por todos rescebido por Rey (CroEsp, 28v)

e. dellos fue luenga mente posseyda (CroEsp, 11r)

f. la qual antigua mente fue $\mathrm{mu}<\mathrm{n}>$ cho preciada en israel (CroEsp, CroEsp,18v)

g. que como fuese por ellos ordenado que (CroEsp, 29r)

h. \& fue asi llamada de vn gran Rio llamdado indo (CroEsp, 3v)

i. $\quad$ es muchas vezes esperimentado (CroEsp,7v)

j. $\quad$ Enesta prouincia fue primero fallada el arte magica (CroEsp, 10v)

k. Enesta fue primero fallado el vso dela purpura (CroEsp, 11r)

1. fue despues llamado iuan yrcano (CroEsp, 13v)

m. delas amazonas es asi llamada (CroEsp, 14r)

n. es asi llamada de aquellas cinco cibdades que (CroEsp, 16r)

o. $\quad$ es [^asy] llamado por que (CroEsp, 16r)

p. Fue asi llamada de dos muy nobles cibdades (CroEsp, 18r)

q. Fue antigua mente llamada trinaclia (CroEsp, 19v)

r. fue antigua mente ganada. por armas por los griegos (CroEsp, 21r).

s. por esso es tan bien llamada barbaria (CroEsp, 21v)

t. fue antigua me $<$ n $>$ te ganada por los griegos (CroEsp, 21v) 
es muy mas poblado (CroEsp, 24r)

x. $\quad$ es muncho poblado (CroEsp, 24v)

y. $\quad$ Fue antigua me $<$ n $>$ te llamada albia (CroEsp, 25r)

v. fue antigua me $<$ n $>$ te llamada goda (CroEsp, 26r)

w. $\quad y$ en tanto fue enel mundo temida que (CroEsp, 26r)

z. $\quad$ es $\mathrm{mu}<\mathrm{n}>$ cho poblada (CroEsp, 30r)

$\alpha$. ASIRIA fue asi llamada de asur (CroEsp, 11r)

$\beta$. fue muncho espantado (CroEsp, 11v)

$\gamma$. MACEDONIA fue antigua me $<$ n $>$ te llamada encia (CroEsp, 13v)

$\delta$. $\quad$ Es asi llamada de vna montąa que (CroEsp, 13v)

\&. $\quad$ ETIOPIA es asi llamada del color delos habitadores della (CroEsp, 16r)

$\zeta$. $\quad$ Esta fue primero llamada canaam (CroEsp, 11r)

$\eta$ ALBANIA es asi llamada por el color dela gente que (CroEsp, 12r)

$\theta$. FENICIA es asi llamada del no $<$ n $>$ bre del searor que (CroEsp, 18r)

t. $\quad$ MEDIA es asi llamada de medo (CroEsp, 18r)

$\kappa$. $\quad$ CHIPRE es asi llamado de vna cibdad q<ue> (CroEsp, 19r)

$\lambda$. germania fue asi llamada a germinando (CroEsp, 20v)

$\mu$. $\quad$ Espaða fue antigua me $<\mathrm{n}>$ te llamada esperia (CroEsp, 21v)

v. DALMACIA es asi llamada de vna gran cibdad q<ue $>$ (CroEsp, 27v)

$\xi$. TRACIA es asi llamada por tiro fijo de iafet (CroEsp, 27v)

o. $\quad$ ACAya es asi llamada por anteo (CroEsp, 28r)

$\pi$. MAVRETANIA del color delos habitadores della es asi llamada (CroEsp, 17r)

p. SAMARIA dela principal cibdad que enella ay es asi llamada (CroEsp, 18v)

५. fuele respondido por su Reyna (CroEsp, 14v)

(18) a. ca el no auia avn bie $<$ n $>$ visto a PieMag (PieMag, 4v)

b. $\quad$ Ca enel mundo yo no he mas amada persona que a el. (PieMag, $7 \mathrm{v}$ )

c. $\quad$ E soy a( )qui venido con poca compaña (PieMag, 11r)

d. E quando ellos ouieron assaz hablado en vno (PieMag, 11v)

e. Quando vino el dia de nuestra señora de mañana que los caualleros auia $<$ n $>$ ya oydo missa (PieMag,13r)

f. Quando ellos ouieron asaz hablado: pierres por prouar a PieMag (PieMag, 15v)

g. $\quad$ E $\mathrm{q}<\mathrm{u}>\mathrm{a}$ 'ndo el ouo a su plazer $\mathrm{co}<\mathrm{n}>\mathrm{te}<\mathrm{m}>$ plado su hermosa cara (PieMag, 17v)

h. $\quad \&$ ouo bien mirado y besado aq $<$ ue $>$ lla ta $<$ n $>$ dulce $\&$ plazie $<$ n $>$ te: pequeña y bermeja boca (PieMag, 17v)

i. $\quad$ E haziendo esto fue tan presto transido de amores (PieMag, 17v)

j. $\quad$ ca contra vos señor dios yo he muy grauemente pecado \& offendido (PieMag, 18v)

k. $\quad$ E qua $<$ n $>$ do ella ouo harto gritado $\&$ andado (PieMag, 20r)

1. $\quad \mathrm{q}<\mathrm{ue}>$ es agora llamada Francia (PieMag, 2r)

m. $\quad$ q $<$ ue $>$ amor al $\mathrm{q}<\mathrm{u}>\mathrm{a}^{`} \mathrm{l} \mathrm{ni}<\mathrm{n}>$ gu $<\mathrm{n}>$ coraço $<\mathrm{n}>$ puede $\mathrm{r}<\mathrm{e}>$ sistir la auia $\mathrm{ta}<\mathrm{n}>$ fuerteme $<$ n $>$ te ferido (PieMag, 6r)

n. $\quad$ y se que el es aqui venido por amor de mi (PieMag, 7r) 
o. $\quad y$ sabed señora que su incomparable hermosura me ha tan fuertemente herido enel coraçon (PieMag, 8r)

p. el qual estaua enla capilla en donde el hauia otras vezes hablado conella (PieMag, 9v)

q. $\quad$ yo he muy gran plazer de que vos soys aqui venido (PieMag, 10v)

r. \& no ayays lo que aueys tan lealmente ganado (PieMag, 11r)

s. $\quad$ q $<$ ue $>$ magalo $<$ n $>$ a seria dello enojada (PieMag, 18r)

t. $\quad$ Cierto si yo me soy tan presto declara[ ]da a vos (PieMag, 20r)

u. Ay de mi maluada $\mathrm{q}<\mathrm{ue}>$ vos he tanto culpado \& injuriado (PieMag, 20v)

x. que nos ha assi tan presto apartado (PieMag, 20v)

y. $\quad$ si no era por fuerça $\mathrm{d}<\mathrm{e}>$ tenido (PieMag, 22r)

v. $\quad$ q $<$ ue $>$ auia muy bie $<$ n $>$ pagado (PieMag, 26r)

w. que yo ouiesse $\mathrm{ta}<\mathrm{n}>$ to dolorosame $<$ n $>$ te perdido mi dulce y leal esposa (PieMag, 26v)

z. $\quad$ el $\mathrm{q}<\mathrm{u}>\mathrm{a} \ 1$ ella auia tantas vezes desseado (PieMag, 28r)

$\alpha$. al qual por vuestra benigna gracia yo era $\operatorname{ta}<\mathrm{n}>$ noblemente desposada (PieMag, 21v)

En las construcciones de infinitivo se han registrado 137 casos con elementos adverbiales interpolados entre el verbo regente y la forma verbal no flexionada. En (19-21) se presentan los 41 casos medievales y en (22-25) los 96 casos que encontramos en el corpus preclásico.

(19) a. $\quad \&$ q $<$ ue $>$ podam $<$ os $>$ firme mient $<$ re $>$ defender $\mathrm{s}<$ us $>$ pueblos. (FueJuz, 10v)

b. $\quad \mathrm{q}<$ ue $>$ ante $\mathrm{q}<\mathrm{ue}>\mathrm{ni}<\mathrm{n}>$ guno aya el regno; ante $\mathrm{p}<$ ro $>$ meta $\mathrm{p}<$ or $>$ sac $<\mathrm{r}>\mathrm{a}{ }^{`} \mathrm{~m}<\mathrm{en}>$ to $\mathrm{d}<\mathrm{e}>\mathrm{g}<\mathrm{u}>\mathrm{a}$ `rdar esta ley. (FueJuz, 11r)

c. en tal man $<$ er $>$ a $\mathrm{q}<\mathrm{ue}>$ las pueda $<\mathrm{n}>$ depues $\mathrm{d}<\mathrm{e}>$ mandar $\mathrm{q}<\mathrm{u}>\mathrm{a}$ `ndo $\mathrm{q}<\mathrm{u}>\mathrm{i}$ isiere $<\mathrm{n}>$. (FueJuz, 11v)

d. $\quad$ ca el muerto $\mathrm{q}<\mathrm{ue}>\mathrm{n}<\mathrm{on}>$ pued $<\mathrm{e}>$ ya entend $<\mathrm{e}>\mathrm{r}$ el castigo (FueJuz, 12r)

e. $\quad$ fu $<$ er $>$ as $t a<$ n $>$ to $q<$ ue $>$ el iuyz puede dos dias en la selmana. o cada dia a ora de medio dia. si $\mathrm{q}<\mathrm{u}>\mathrm{i}$ sier folgar en su casa. \& $\mathrm{n}<\mathrm{on}>\mathrm{au}<\mathrm{er}>\mathrm{p}<\mathrm{le}>$ yto en todo el ot $<\mathrm{r}>\mathrm{o}^{`}$ tiempo. (FueJuz, 14r)

f. Del iuyz $\mathrm{q}<\mathrm{ue}>\mathrm{q}<\mathrm{u}>\mathrm{i}$ er bien ente $<\mathrm{n}>$ der el pleyto. (FueJuz, 14v)

g. $\quad$ Al iuyz si algu $<$ n $>$ o demanda razo $<$ n $>$. delo $\mathrm{q}<$ ue $>$ iulgo. o ant $<$ e $>$ ot $<\mathrm{r}>\mathrm{o}$ ioyz. o ant $<\mathrm{e}>\mathrm{q}<\mathrm{u}>\mathrm{i}$ 'en mandare el Rey. deuel ende respo $<$ n $>$ der. (FueJuz, 16r)

h. deue $\mathrm{p}<\mathrm{r}>\mathrm{i}$ mera mientre saber la $\mathrm{u}<\mathrm{er}>$ dat delos testimonios si los ouier en el pleyto. (FueJuz, 14v)

i. $\quad \&$ por end $<$ e $>$ deuem $<$ os $>$ p $<$ r $>$ i m $<$ er $>$ a mientre ordenar $1<0$ os $>$ $\mathrm{p}<\mathrm{ro}>$ uechos de los $\mathrm{p}<\mathrm{r}>\mathrm{i}$ ncipes. (FueJuz, 10v)

j. $\quad$ mas esto puede $<$ n $>$ bien faz $<$ er $>$ q $<$ ue $>$ ant $<$ e $>$ q $<$ ue $>$ aq $<$ ue $>1$ las testimonias $\mathrm{q}<\mathrm{ue}>$ fuero $<\mathrm{n}>$ recebidas sea $<\mathrm{n}>$ muertas. (FueJuz, 15r)

k. $\quad \mathrm{q}<$ ue $>$ deue $\mathrm{p}<\mathrm{r}>\mathrm{i}$ mera mientre fazer (FueJuz, 14v) 
1. $\quad$ si el iuyz a $\mathrm{q}<\mathrm{u}>\mathrm{i}$ en ruega $<\mathrm{n}>\mathrm{q}<\mathrm{ue}>$ faga Justicia quier depues oyr el pleyto \& falar por $\mathrm{u}<$ er $>$ dat $\mathrm{q}<\mathrm{ue}>\mathrm{aq}<\mathrm{ue}>\mathrm{l} \mathrm{q}<\mathrm{ue}>$ se $\mathrm{q}<\mathrm{ue}>$ rello $\mathrm{p}<\mathrm{r}>$ im $<$ er $>$ a mientre $\mathrm{q}<\mathrm{ue}>$ se $\mathrm{q}<\mathrm{ue}>$ rello $\mathrm{c}<\mathrm{on}>$ tuerto. (FueJuz, 18v)

(20) a. si non fiziessen un cas tiello de fuste tan alto que pudiessen del tirar enla villa piedras \& saetas \& que (ConUlt, 1v)

b. $\quad$ assi que pudiessen del uer toda la çipdat. \& tirar poro quisiessen piedras. \& saetas. (ConUlt, 2r)

c. \& non osauan ya sobir alos muros (ConUlt, 2r)

d. \& començaron luego a combater la çipdat. mas fuerte que (ConUlt, 2r)

e. \& esforçaron mas de yr contra so prelado. (ConUlt, 3v)

f. que saliessen otro dia con gra $<\mathrm{n}>$ t procession a reçebirle. (ConUlt, $5 \mathrm{r}$ )

g. \& fizo y uenir todos los prelados del tierra (ConUlt, 6r) (S)

h. $\quad$ mas el $n<$ on $>$ quiso y uenir. (ConUlt, $7 \mathrm{v}$ )

i. $\quad$ por $\mathrm{q}<\mathrm{ue}>\mathrm{q}<\mathrm{ue}>$ rie luego que uiniesse el tiempo del uerano. fazer algu $<\mathrm{n}>$ fech $<\mathrm{o}>\tan$ grant en la tierra de. Suria (ConUlt, 10v)

j. $\quad$ E por aquello querie alli tener el yuierno. (ConUlt, 10r)

k. començo luego a subir el poçon de la yerua por el braço (ConUlt, 11r)

1. Et mando luego uenir ante si. los de su $l i<n>$ nage que estauan y. \& todos los omnes buenos de la hueste (ConUlt, 11v)(S)

m. aq<ue $>1$ punnaua $\mathrm{q}<\mathrm{ua}>$ nto podie en ayudar al fijo mayor. que fuesse Emperador (ConUlt, 12r)

n. por que non podien ya assi salir. (ConUlt, 13v)

o. que non osaua $<\mathrm{n}>$ ya salir. a ningun cabo. (ConUlt, $14 \mathrm{r}$ )

p. assi que non osauan ya salir a ningun cabo.(ConUlt, 14rbis)

q. ni<n $>$ aun los labradores non osauan salir a labrar. nin a sembrar. (ConUlt, 14r)

r. La Reyna fizo y fazer una torre muy fuerte \& muy buena \& muy alta. (ConUlt, 14v)

s. $\quad$ aquella Reyn $<$ a $>$ melissen. amaua mucho seruir a nuestro $\mathrm{se}<$ n $>$ nor dios (ConUlt, 15v)

t. uio assi estar al Rey (ConUlt, 15v)

u. $\quad$ E punnaua mas de tener se uicioso que (ConUlt, 17v)

x. $\quad$ Et fizo luego fazer engennios muy buenos. (ConUlt, 18r)

y. Ot $<\mathrm{r}>0$ `ssi los arqueros \& los ballesteros non quedaua $<\mathrm{n}>$ poco $\operatorname{nin}$ mucho de $c 0<$ n $>$ bat $<$ er $>$ aq $<$ ue $>$ llos que se parauan por los muros $\&$ por las torres.(ConUlt, 18r)

v. $\quad$ Et mando luego cauar una piesça del muro. (ConUlt, 19r)

w. fuero $<\mathrm{n}>$ se pora los algibes a sacar del agua. (ConUlt, 23v)

z. $\quad$ ca ya no $<$ n $>$ lo podien mas soffrir (ConUlt, 25v)

$\alpha$. $\quad$ E aquel no $<$ n $>$ pudo ya soffrir. la loçania. ni $<$ n $>$ la soberuia daquellos Ricos omnes. (ConUlt, 26v)

$\beta$. $\quad$ Et $m a<n>$ do luego llamar a un cauall $<$ er $>$ o que sabie muy bien el arauigo (ConUlt, 26r)

$\gamma$. $\quad$ E aquellos se trabaiauan mas que todos los otros de faz<er $>$ mal. a los $\mathrm{xp}<$ ist $>$ ianos (ConUlt, 26v) 
(21) a. \& fizo luego derribar todas las torres del muro bien fasta la meatad.

(InfLara, 39r)

b. fueron luego ferir enlos moros (InfLara, 37v)

c. \& enbio luego dezir a don gonçalo gustios que (InfLara, 34r)

d. nunca los podredes ya cobrar (InfLara, 38v)

e. Mas agora vos dexaremos aquj de fablar desto (InfLara, 38v)

f. Muño salido come $<\mathrm{n}>$ ço estonçes de los esforçar (InfLara, 36v)

g. $\quad$ por que yo no $<$ n $>$ me puedo aca vengar dellos en tierra de $\mathrm{xp}<$ ist $>$ ianos (InfLara, 34v)

h. que non sse les podien ya defender (InfLara, 37r)

i. $\quad$ si las podras por auentura conosçer (InfLara, 38r)

j. $\quad$ ca sse les non podien ya anparar (InfLara, 37v) actos (OrdSev, 5r)

b. $\quad$ por $\mathrm{q}<\mathrm{ue}>\mathrm{no}<\mathrm{n}>$ se pueden enellas mantener (OrdSev, 6v)

c. por ende queriendo sobre esto proueer ordenamos \& mandamos que (OrdSev, 6v)

d. $\quad$ Por ende $\mathrm{q}<\mathrm{ue}>$ riendo enesto proueer (OrdSev, 8r)

e. por $\mathrm{q}<\mathrm{ue}>\mathrm{p}<\mathrm{er}>$ sona alguna no $<\mathrm{n}>$ pueda della pretender ignorancia (OrdSev, 7r)

f. $\quad \&$ que los alcaldes mayores della no podia $<$ n $>$ asi administrar la iustiçia (OrdSev, 9r)

g. $\quad$ que pueda $<\mathrm{n}>\&$ sepa $<\mathrm{n}>$ bien administrar iustiçia $($ OrdSev, $11 \mathrm{r})$

(23) a. que por amor dela vida eterna no recelan en biuas llamas de fuego quemarse (CroEsp, 4v)

b. que ni<n $>$ gun onbre podria alli entrar (CroEsp, 10v)

c. que non se pueden bie $<\mathrm{n}>$ deuisar (CroEsp,11v)

d. $\quad \mathrm{q}<$ ue $>$ quieras $\mathrm{co}<\mathrm{n}>$ mugeres $\mathrm{co}<\mathrm{n}>$ tender $($ CroEsp, $14 \mathrm{v})$

e. lo cual parescio enlos oios delos mortales ser inposible tan fuerte cibdad poder destruyrse (CroEsp, 15v)

f. determino de iamas boluer a el \& de buscar nueua tierra (CroEsp,28r)

g. que ose contra ellos pelear (CroEsp, $9 \mathrm{v})$

h. E iulio cesar (...) temio enesta prouincia de entrar (CroEsp, 26r)

i. quien querra en suma alguna parte de aquellas saber (CroEsp,28v)

j. $\quad$ segun lo pude meior colegir delos auctores (CroEsp, 28v)

k. De napol (...) no co<n>uiene mas dezir (CroEsp, 29v)

(24) a. no puede mas valer (PieMag, 3r)

b. $\quad$ y se fue derechamente a posar en vna plaça (PieMag, 3v)

c. $\quad$ E q $<\mathrm{u}>\mathrm{a}$ `ndo pierres oyo asi fablar a PieMag (PieMag, 5r)

d. a mi no basta tan solamente dar gracias al rey (PieMag, 5r)

e. $\quad \&$ no le $\mathrm{q}<\mathrm{u}>$ i $`$ so mas $\mathrm{p}<\mathrm{re}>$ gu $<$ n $>$ tar dello (PieMag, 5v)

f. yo desseo mucho saber su linaje (PieMag, 6r)

g. ca ella lo desea mucho saber (PieMag, 6v) 
h. $\quad$ quando Pierres oyo assi hablar la ama (PieMag, 6v)

i. $\quad$ Qua $<$ n $>$ do la ama oyo assi hablar a PieMag (PieMag, 7v)

j. $\quad$ E quando PieMag oyo a su ama no lo pudo mas suffrir \& dixo le (PieMag, 7v)

k. Entonces la ama conosciendo su voluntad no le quiso mas contradezir (PieMag, 7v)

1. E quando PieMag oyo tan subitame $<$ n $>$ te hablar a su ama (PieMag, 7v)

m. \& por esto yo querria bie $<$ n $>$ saber quie $<$ n $>$ es (PieMag, 8r)

n. Que sin falta yo no $\mathrm{q}<\mathrm{u}>\mathrm{i}$ ero mas tardar que yo no fable conel. (PieMag, 9r)

o. $\quad$ E vos dessea mucho ver y hablar co<n $>$ vos. (PieMag, $9 v$ )

p. yo puedo bien dezir que (PieMag, 10r)

q. $\quad \&$ torno otra vez a besar a Pierres muy dulcemente (PieMag, 11v)

r. el començo fuerteme $<\mathrm{n}>$ te a pensar (PieMag, 12v)

s. $\quad \mathrm{q}<\mathrm{u}>\mathrm{i}$ ero eneste dia mostrar mi fuerça y proeza (PieMag, 13v)

t. $\quad$ Ca el coraço $<$ n $>$ no le podia mas sofrir (PieMag, 13v)

u. $\quad \&$ no $\mathrm{q}<\mathrm{u}>\mathrm{i}$ so en ninguna manera ferir a su tio don Jayme (PieMag, 14r)

x. $\quad$ como el sabia muy bien fazer (PieMag, 14v)

y. \& no quiso mas tornar ala justa (PieMag, 14v)

v. Ca yo puedo bien dezir (PieMag, 14v)

w. Quando pierres oyo tan piadosame $<$ n $>$ te fablar a PieMag (PieMag, 15v)

z. yo he deliberado de nunca partir desta tierra (PieMag, 16r)

$\alpha$. E yo querria antes morir que vos dexar (PieMag, 16r)

$\beta$. $\quad$ Y [ ]la linda Magalo $<\mathrm{n}>$ a lo auia alli de esperar. (PieMag, 16r)

$\gamma$. Quando los caualleros entendieron al Rey ellos se fueron luego a armar (PieMag, 17r)

$\delta$. Dexemos agora de hablar del rey (PieMag, 17r)

$\zeta$. ca su noble coraçon no podia mas suffrir el gran dolor que auia (PieMag, 18v)

$\eta$. \& començo muy fuerteme $<$ n $>$ te a llorar \& an-dar por el monte (PieMag, 20r)

$\theta$. Ay fortuna tu no comie $<\mathrm{n}>$ ças agora de perseguir los buenos y leales. (PieMag, 20v)

1. $\quad \mathrm{Y}$ dexad me por vuestro dulçor ver antes que yo muera mi señor \& mi marido. (PieMag, 20v)

$\kappa$ v. vos no deueys por esso burlar delos pobres de jesu $x p<i s t>0$ (PieMag, 21r)

n. $\quad$ y la hizo aq<ue>lla noche dormir \& reposar conella. (PieMag, 22v)

$\mu$. PieMag se puso con gran deuocion a seruir los dolientes (PieMag, 3r)

v. Quando PieMag entendio todas estas palabras ella començo fuertemente a llorar (PieMag, 24r)

$\xi$. Dexemos agora de hablar de PieMag (PieMag, 24v)

o. Por esto señor plega vos liberalmente contentar de mi partida (PieMag, 25r) 
$\pi$. Dexemos agora de hablar del conde y dela co $<$ n $>$ dessa y de PieMag (PieMag, 26v)

ค. $\quad \&$ començo muy fuertemente a llamar \& dar bozes (PieMag, 26v)

$\sigma . \quad \&$ començo amargamente a llorar (PieMag, 26v)

$\tau$. començo a andar por la villa a[ ]fin que mas ayna pudiesse sanar (PieMag, 27r)

v. començo muy bien de sanar (PieMag, 27v)

$\varphi$. $\quad$ quando ella oyo ta $<\mathrm{n}>$ fuertemente sospirar a pierres (PieMag, 28r)

$\chi$. Quando PieMag oyo assi hablar $\mathrm{d}<\mathrm{e}>$ fortuna (PieMag, 28r)

$\psi$. $\quad$ ella lo començo muy dulcemente a consolar (PieMag, 28r)

$\chi$. Qua $<$ n $>$ do el conde \& la co $<$ n $>$ dessa oyeron assi fablar la hospitalera (PieMag, 29v)

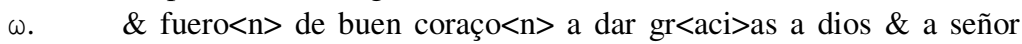
sant pedro (PieMag, 30v)

A. Y podreys dello mas valer. (PieMag, 2v)

B. quando el noble cauallero Pierres entendio assi hablar el cauallero (PieMag,2v)

C. que no sabria ho $<\mathrm{m}>$ bre deuisar ni nombrar manera de vianda que (PieMag, 5r)

D. por lo $\mathrm{q}<\mathrm{u}>\mathrm{a}^{`} \mathrm{l}$ venid vos muchas vezes a tomar plazer enesta casa (PieMag, 5r)

E. $\quad$ obliga $<$ n $>$ do me $\mathrm{p}<$ ar $>$ a sie $<$ m $>$ pre jamas de ser vuestro humilde seruidor (PieMag, 5r)

F. $\quad$ si por ve $<$ n $>$ tura el fuesse de gra $<$ n $>$ linaje \& noble $\mathrm{q}<$ ue $>$ ella lo $\mathrm{q}<$ ue $>$ rria mas amar $\mathrm{q}<\mathrm{ue}>$ a $\mathrm{p}<$ er $>$ sona $\mathrm{d}<\mathrm{e}>1 \mathrm{mu}<\mathrm{n}>$ do

(PieMag, 5v)

G. creed que enel mundo no me sabriades cosa mandar (PieMag, 6r)

H. Qua $<$ n $>$ do la ama oyo assi hablar a PieMag (PieMag, 7v)

I. que yo no lo puedo mas sufrir ni encubrir (PieMag, 8r)

J. $\quad$ Y señora el hecho es tal que el dessea sobre todas las cosas del mundo hablar con vos en secreto. (PieMag, 9r)

K. que yo no podria jamas auer[ ]vuestra buena gracia (PieMag, 11r)

L. $\quad$ E qua $<$ n $>$ do el veya que podia sin peligro hartar su noble coraçon (PieMag, 12v)

M. a[ ]fin que el pudiesse mejor conq $<$ u $>$ i $` s t a r$ la gracia \& amor de PieMag (PieMag, 12v)

N. ala $\mathrm{q}<\mathrm{u}>\mathrm{a} \backslash \mathrm{l}$ el $\mathrm{q}<\mathrm{ue}>$ ria mas $\mathrm{co}<\mathrm{m}>$ plazer $\mathrm{q}<\mathrm{ue}>$ a $\mathrm{p}<\mathrm{er}>$ sona del mundo (PieMag, 18r)

O. que no son ciertas sie $<\mathrm{m}>$ pre las deue hombre auer en esperança (PieMag, 24r)

(25) a. qujerome Agora estar vn poco (Celestina, 95r)

b. $\quad \mathrm{qu}\left(\wedge^{\wedge}\right) \mathrm{eje}$ no se sabe A la Razon Someter/(Celestina, 96v)

c. que entravan allj allorar sus' pecados' (Celestina, 100r) 
La interpolación del sujeto -forzada en las formas verbales compuestas con participio y algo dudosa en las construcciones de infinitivo del español actual-, aunque con menor frecuencia, se documenta en los textos antiguos analizados. Se han recogido 25 casos con sujeto interpolado entre el verbo regente y el participio: 12 en el corpus medieval y 13 en el corpus preclásico; los ejemplos medievales se presentan en (26-28), los preclásicos en (29-31).

(26) a. fueras si era el pl<e>ito començado. (FueJuz, 12v)

b. $\quad$ si al iuyz fuere $\mathrm{p}<$ ro $>$ uado o al obispo $\mathrm{q}<\mathrm{ue}>$ iulgo tuerto lo que mando a tomar a aq $<$ ue $>1$ a $q<$ ue $>$ lo iulgaro $<$ n $>$ sea todo entregado (FueJuz, 14v)

(27) a. Teniendo el çercada aquella çipdat (ConUlt, 20v)

b. $\quad$ E por que estau<a $>$ aquel castiello aredrado una piesça de 1(o)[a]s ot $<$ ra $>$ s fortalezas de los $\mathrm{xp}<$ ist $>$ ianos (ConUlt, 14v)

c. que serien todos culpados enla trayçion. (ConUlt, 22v)

d. Quando los prelados fueron todos ayuntados en la egl<es $>$ ia de sant Pedro de antiocha. (ConUlt, 6v)

e. que siempre serie Rey muy auenturado. (ConUlt, 20r)

f. ca eran ya todos semurziados del fuego \& del fumo. (ConUlt, 25v)

g. que el ayre era corronpido. \& toda la $\mathrm{t}<\mathrm{ier}>\mathrm{ra}$ cubierta dellas. (ConUlt, 23v)

(28) a. los caualleros \& los` parientes \& los amigos de Ruy blasq $<$ ue $>$ s fueron alli todos llegados (InfLara, 33r)

b. $\quad$ Despues que la carta ouo el moro fecha (InfLara, 34v)

c. \& fueron los $\mathrm{xp}<\mathrm{ist}>$ ianos vençidos (InfLara, 38r)

(29) a. que muy presta me $<$ n $>$ te fue todo acabado (CroEsp, 31v)

b. en tie $<\mathrm{n}>$ po del qual fue dardania primera vez destruyda (CroEsp,12v/13r)

c. $\quad$ Es la piel suya pintada de diuersas colores (CroEsp, 7r)

d. fue la vna llamada marsepia. La otra laupato (CroEsp, 14r)

e. $\quad$ es esta prouin cia de toda parte cercada de mar (CroEsp, 19r)

f. es zelanda muy poblada (CroEsp, 23r)

(30) a. en auer yo hallado vn tan noble cauallero (PieMag, 11r)

b. Ay mi amigo pierres mi amor \& mi esperança y como vos he yo perdido (PieMag, 20r)

c. Ay mi señor en que vos he yo errado (PieMag, 20r)

d. Ay mi dulce amigo Pierres aueys vos visto en my cosa que (PieMag, 20r)

e. porque no so yo muerta con vos (PieMag, 20v)

(31) a. que ... A dios' tengo yo ofresçido (Celestina, 94r)

b. pocas mataduras has tu vis'to enla barriga (Celestina, 98r) 
La interpolación del sujeto se documenta 42 veces en las construcciones de infinitivo: 32 veces en los textos medievales y 10 veces en los preclásicos; los casos medievales se presentan en (32-34), los preclásicos en (34-35). Nótese que en abundantes casos el sujeto intercalado es impersonal, representado por el sustantivo omne -véanse (32a-c), (32e-f), (33o), (33q) -o su forma moderna hombre - como en (35d) y (36c)-.

(32) a. \& por $\mathrm{q}<\mathrm{ue}>\mathrm{aq}<\mathrm{ue}>$ sto pueda $\mathrm{o}<\mathrm{mn}>\mathrm{e}$ entender si gelo da por forcia. o por engan $<\mathrm{n}>\mathrm{o}$ del $\mathrm{p}<\mathrm{r}>\mathrm{i}$ ncipe. (FueJuz, 10v)

b. $\quad \&$ si pudier $\mathrm{o}<\mathrm{mn}>$ e entender $\mathrm{q}<\mathrm{ue}>$ gelo da $\mathrm{co}<\mathrm{n}>$ su uolu $<$ n $>$ tat. o gelo diesse el p<r>i`ncipe. (FueJuz, 10v)

c. $\quad \&$ desto lo puede $0<\mathrm{mn}>\mathrm{e}$ mais entender. por $\mathrm{q}<\mathrm{ue}>$ (FueJuz, 11v)

d. Ot $<$ r $>$ o `si no $<$ n $>$ q $<$ ue $>$ rem $<$ os $>$ nos sofrir $\mathrm{q}<$ ue $>$ ne $<$ n $>$ guno nol ponga culpa falsa mient $<\mathrm{r}>\mathrm{e}^{`}$. ni $<\mathrm{n}>$ lo maldiga. (FueJuz, 11r)

e. $\quad \mathrm{q}<\mathrm{ue}>$ las $\mathrm{n}<\mathrm{on}>$ puede $\mathrm{om}<\mathrm{n}>\mathrm{e}$ ordenar ca algunas cosas fortes son ordenadas. p<or $>$ escuras palabras. (FueJuz, 11r)

f. $\quad$ Et otrossi deue om $<\mathrm{n}>\mathrm{e}$ acatar $\mathrm{q}<\mathrm{u}>$ a`nto mas fuere $\mathrm{p}<\mathrm{er}>$ longado. (FueJuz, 14r)

la partida $\mathrm{q}<\mathrm{ue}>$ deue $<$ n $>$ amas las partes auer deue $<$ n $>$ pagar el ioyz. \& el sayon. (FueJuz, 15v)

g. $\quad$ ca lo no $<$ n $>$ deue $<$ n $>$ todos razonar de so uno de ambas las partes. solamientre (FueJuz, 17r)

h. deue cada uno pechar al Rey tanto $\mathrm{q}<\mathrm{u}>\mathrm{a}$ `nto era la $\operatorname{dema}<\mathrm{n}>$ da. (FueJuz, 17v)

i. $\quad \mathrm{n}<$ on $>$ deue el iuyz faz $<$ er $>$ major he $<$ n $>$ mienda (FueJuz, 18v)

(33) a. por contar $c 0<m>$ mo uinieron el princep de antiocha. \& el conde de Tipre a la çerca de belinas a ayudar al Rey de ierusale $<\mathrm{m}>\&$ alos de domas. (ConUlt, 1v)

b. de manera que non podie ninguno andar por la uilla (ConUlt, 2r)

c. Mas agora dexa aq $<\mathrm{u}>\mathrm{i}$ la ystoria de fablar desto (ConUlt, 1v)

d. Mas agora dexa aqui la estoria. a fablar desto (ConUlt, 2v)

e. $\quad$ E punno entonçes el prinçep de saber del legado que uoluntad tenie contral patriarca de antiocha (ConUlt, $3 \mathrm{v}$ )

f. Mas agora dexa aqui la estoria a fablar del (ConUlt, 8v)

g. $\quad$ quel enuiaua el Rey mucho saludar. co $<$ m $>$ mo amigo. (ConUlt, 10v)

h. $\quad$ q<ue $>$ pudiesse el auer a su mug<ier $>$ (ConUlt, 11r)

i. $\quad$ Agora dexa aqui la ystori<a a fablar desto. (ConUlt, 12v)

j. $\quad$ quiso se el tener con la mayor parte. (ConUlt, 12v)

k. Mas agora dexa aq<u>i` la ystoria a contar del (ConUlt, 16r)

1. E alos Ricos omnes loçanos que por su soberuia querien quebrantar a sus uezinos fazie los ella uenir a derecho de guisa que (ConUlt, 17r)

m. $\quad c o<m>$ mo fue el Rey de ih $<$ e $>$ r $<$ usa $>1<$ e $>$ m con su hues te a reçebir la mayor çipdat de arauia. (ConUlt, 21r)

n. $\quad$ E aq $<$ ue $>$ llo enbiaua el dezir al Rey por $\mathrm{e}<$ n $>$ ganno. (ConUlt, 21v) 
o. aquella ora deue omne demandar ayuda \& acorro a $n<$ uest $>$ ro se $<$ n $>$ nor dios. (ConUlt, $5 \mathrm{v}$ )

p. començaron todos a llorar con grant allegria. (ConUlt, 25v)

q. $\quad$ por que deue omne auer toda uia mala sospecha. del seruiçio de su enemigo (ConUlt, 27r)

(34) a. vna ssemana antes `ue las bodas sse acabasen mando don Rodrigo alçar vn tablado en la glera allende el Rio (InfLara, 32r)

b. Estonçes enbio Ruy blasq<ue $>$ s dezir por toda la tierra que (InfLara, 35r)

c. ca de quantos alli fueron a alançar nunca ninguno pudo dar en somo del tablado ssi non el (InfLara, 32v)

d. $\quad \mathrm{q}<\mathrm{ue}>\mathrm{q}<\mathrm{u}>\mathrm{i}$ 'ero yo ferir luego en aquesta az $\mathrm{p}<\mathrm{r}>\mathrm{i}$ mera (InfLara, 36v)

e. que le fagades vos luego descabeçar (InfLara, 34v)

f. $\quad$ Entonçes enbiaron los Jnfantes a pedir treguas a uiara (InfLara, 37r)

(35) a. E acabando el conde de hablar dixo la condessa (PieMag, 2v)

b. como por amor della yua $<$ n $>$ muchos buenos caualleros a Napoles a hazer justas (PieMag, 2r)

c. $\quad$ señora ante pueda yo morir de mala muerte $\mathrm{q}<\mathrm{ue}>$ (PieMag, $8 \mathrm{v}$ )

d. que vos deue hombre creer. (PieMag, 10r)

e. $\quad$ O maluado q<ue $>$ so yo porq<ue $>$ me quiero yo matar (PieMag, 18v)

f. $\quad$ Ay señor pierres y que pudiera yo mas hazer por vos (PieMag, 20r)

g. $\quad \mathrm{y}$ despues pensaua que podria ella hazer (PieMag, 20v)

(36) a. dexa la tinjebra Aconpañar Al triste y al desdich( )ado la ceguedad (Celestina, 94v)

b. $\quad$ que no puede mj paçiençia tolerar (Celestina, 94v)

c. peor estremo es' dexars'e onbre caer de s'u meres[*çimjento] que (Celestina, 97r)

La intercalación de complementos regidos se ha registrado 4 veces en los textos analizados; 3 veces se documenta en la parte medieval y una sola vez en la parte preclásica del corpus; cfr. (37-38)

(37) a. ouieran al legado sacado de la cipdat a grant desondra (ConUlt, 7v)

b. $\quad$ E pues que lo ouo todo fecho muy bie $<$ n $>$. \& co $<$ m $>$ plidamientre (ConUlt, 14v)

c. Despues que los Jnfantes ouieron aquel om<n>e muerto (InfLara, $33 \mathrm{v})$

Pues assi es que esta noble dama ha tanto hecho por vos (PieMag, $30 v)$

La intercalación de constituyentes regidos es bastante más frecuente en las construcciones de infinitivo: se ha registrado 24 veces en todo el corpus; 12 veces 
ocurre en los textos medievales y 12 veces en los textos preclásicos, así como se ve en los ejemplos aducidos en (39-41) y (42-44), respectivamente.

(39) a. otrosi de aq $<$ ue $>$ llas cosas q $<$ ue $>$ ganaro $<$ n $>$ ant $<$ e $>$ q $<$ ue $>$ fuessen reyes. \& $\mathrm{q}<$ ue $>$ era $<$ n $>$ suyas $\mathrm{p}<\mathrm{ro}>\mathrm{p}<\mathrm{r}>\mathrm{i}$ as puede $<\mathrm{n}>$ dellas fazer lo $\mathrm{q}<\mathrm{ue}>\mathrm{q}<\mathrm{u}>\mathrm{i}$ `siere $<\mathrm{n}>$. (FueJuz 11r)

b. $\quad$ si aq<ue $>1$ q<ue $>$ relloso pudier esto mostrar con testimonios (FueJuz 14r)

c. $\quad$ el iuyz lo deue todo entregar todo delo suyo. (FueJuz, 14v)

d. el ioyz deue las testimonias reçebir. (FueJuz, 15r)

e. \& si las pudier todas desdezir fasta $\mathrm{q}<\mathrm{ue}>\mathrm{n}<\mathrm{on}>$ finq $<\mathrm{ue}>$ dos testimonias bonas. (FueJuz, 15r)

f. $\quad \&$ el recabdo $\mathrm{q}<\mathrm{ue}>$ tomo el iuyz delas partes por su no $<\mathrm{m}>$ bre si lo pierde algu $<\mathrm{n}>\mathrm{a}$ [delas] $\mathrm{p}<$ ar $>$ tes $\mathrm{n}<\mathrm{on}>$ lo deue todo ganar el iuyz. ni el sayon. (FueJuz, 17v)

g. $\quad \&$ el recabdo $\mathrm{q}<$ ue $>$ tomo el iuyz delas partes por su no $<\mathrm{m}>$ bre si lo pierde algu $<\mathrm{n}>\mathrm{a}$ [delas] $\mathrm{p}<$ ar $>$ tes $\mathrm{n}<\mathrm{on}>$ lo deue todo ganar el iuyz. ni el sayon. (FueJuz, 17v)

(40) a. enuiaron sus $m a<n>$ daderos \& sus cartas al Califa de egipto. so se $<$ n $>$ nor que era omne muy poderoso. a mostrar le $c o<m>$ mo (ConUlt, 14r)

b. $\quad$ E segunt dize el euang[e]lio yua el a aquel castiello muchas uezes posar. (ConUlt, 14v)

c. $\quad$ quando uiero $<\mathrm{n}>\mathrm{aq}<\mathrm{ue}>$ ll duelo tan grant fazer. (ConUlt, 26v)

(41) a. quando le oyo aq<ue>llo dezir (InfLara, 36v)

b. \& non le dexaron y mas fazer (InfLara, 38r)

(42) a. Como yo sea mortal podre a vos otros dar inmortalidad. (CroEsp, 4r)

b. que el grande alexandre recelo con ella co $<$ n $>$ tender (CroEsp, 26r)

c. quien querra en suma alguna parte de aquellas saber (CroEsp, 28v)

d. que con gran trabaio se puede a ella llegar (CroEsp, 28r)

e. $\quad$ q $<$ ue $>$ quieras co $<$ n $>$ mugeres co $<$ n $>$ tender $($ CroEsp, 14v)

f. mostroles senbrar trigo \& ceuada $\&$ plantar viñas \& politica mente biuir (CroGer, 28v)

(43) a. si vos me quereys algo dello declarar yo gelo hare saber (PieMag, 6v)

b. $\quad$ Y qua $<$ n $>$ do el solda $<$ n $>$ vio a Pierres ta $<$ n $>$ humilmente suplicar le dixo (PieMag, 25r)

c. $\quad y$ entonces vierades caualleros y damas y toda manera de ge $<$ n $>$ te fazer fiestas a Pierres (PieMag, 30r)

(44) a. tener con quien puedan s'us cuytas llorar (Celestina, 95r)

b. $\quad q u(\wedge e) j e n$ no se sabe A la Razon Someter/ (Celestina, 96v)

c. no pueden al dezir (Celestina, 99r) 


\section{Resumen y conclusiones}

Desde luego, la extensión limitada del corpus utilizado no nos permite formular afirmaciones demasiado generalizadas en relación a la evolución de la estructura de las construcciones de participio y de infinitivo, pero en base al análisis expuesto se pueden esbozar algunas conclusiones. El estudio común de los dos tipos de construcciones confirma los datos referentes a las construcciones de participio de estudios precedentes, y proporciona datos parecidos en el caso de las construcciones de infinitivo. Las proporciones semejantes referentes a los dos fenómenos analizados indican que éstos, a grandes rasgos, pueden deberse a las mismas causas en ambos tipos de construcciones. En cuanto a la proporción de las ocurrencias de los dos fenómenos en los textos medievales y en los preclásicos, podemos observar que ésta sorprendentemente- aumenta en el caso de ambos tipos de construcciones en algunos textos preclásicos. Sin embargo, mientras que las proporciones -bajas- de estos fenómenos son paralelas en los textos medievales, su crecimiento aparece en una forma bastante desordenada en los textos preclásicos. Estos datos pueden indicar que probablemente no se trata de la evolución espontánea del sistema lingüístico, sino más bien de fenómenos del estilo latinizante, frecuente en la literatura de la época. Por lo que se refiere a la frecuencia de los dos fenómenos heredados del español arcaico en los diferentes tipos de textos, según los datos de las investigaciones su uso se prefirió más -ya en el período medieval- en obras literarias, mientras que fue bastante raro en los textos de carácter legal. Esta diferencia entre los tipos de textos puede ser explicada por dos factores: el contenido y el valor estilístico. Por una parte, la anteposición de elementos al verbo puede ser relacionada con diferentes funciones discursivas, con cambios de puntos de vista sucesivos, frecuentes en textos narrativos, pero ausentes en textos jurídicos o legales. Por otra parte, la coexistencia de las alternativas sintácticas arcaicas con las actuales en las obras literarias también puede ser un medio apropiado para alcanzar un valor estilístico más alto, el cual no es importante en los textos legales, redactados con objetivos bien distintos. Por supuesto, sólo el análisis de un corpus más amplio puede comprobar la validez de estas primeras explicaciones sugeridas. 